\title{
Principaux fruits de cueillette consommés et commercialisés en Guinée
}

Madina N'Diaye $^{a \star}$, Fodé Bangaly Kéita ${ }^{b}$, Philippe Martinc

${ }^{a}$ CRA, Foulaya, BP 156, Kindia, Guinée

${ }^{\mathrm{b}}$ Direction nationale de l'agriculture, BP 567,

Conakry, Guinée

${ }^{c}$ Cirad-Flhor, 22 BP 874, Abidjan 22, Côte d'Ivoire

${ }^{*}$ Correspondance et tirés à part

Reçu le 10 avril 2002

Accepté le 2 septembre 2002

Fruits, 2003, vol. 58, p. 99-116 (C) 2003 Cirad/EDP Sciences All rights reserved

DOI: 10.1051/fruits:2002040

RESUMEN ESPAÑOL, p. 116

\section{Principal fruits of gathering consumed and marketed in Guinea.}

Abstract - Introduction. Guinea is a country of West Africa with a tropical climate, a hilly relief and a significant hydrographic network. It presents four natural areas in which several fruit-bearing species develop spontaneously. Many of the fruits thus gathered are currently consumed and sold in the national markets. For better exploitation of these products, our investigations tried to constitute a base of reliable data identifying the implied fruit-bearing species, their zones and periods of production, the marketing channel for their products, and their conservation, processing and consumption techniques. Materials and methods. A follow-up of the prices of the fruits gathered and marketed in Guinea and a thorough analysis of their production and use were carried out from March 1998 to February 2000 on two similar markets of the country. For that, necessary information (price, period, packaging, marketing channel) was collected by investigations carried out every fifteen days and consigned into a specific form. Results. The study underlined possible problems of the market supply related to its location. Among the identified species, twelve appeared more important than the others and were studied more. Their production zones were defined. The majority of the fruits marketed on the surveyed markets mature between March and June; they represent a source of income for many people. The techniques of conservation, marketing, processing and consumption were indexed. Conclusion. The survey carried out results in encouraging the protection of the indigenous fruit-bearing species and in advising their progressive introduction into the family orchards. The national research programs also aim at intervening in the processing and conservation of the products resulting from gatherings, which raise large difficulties with the producers.

Guinea / endemic species / fruit crops / picking / production location / market prices / uses

\section{Principaux fruits de cueillette consommés et commercialisés en Guinée.}

Résumé - Introduction. Pays d'Afrique de l'Ouest, la Guinée a un climat tropical et un relief accidenté, avec un réseau hydrographique important. Elle présente quatre régions naturelles dans lesquelles se développent spontanément plusieurs espèces fruitières dont les fruits sont actuellement consommés et vendus dans les marchés nationaux. Afin de mieux exploiter ces produits, des enquêtes ont cherché à constituer une base de données fiables identifiant les espèces fruitières impliquées, leurs zones et leurs périodes de production, le circuit de commercialisation des produits, leurs techniques de conservation, de transformation et de consommation. Matériel et méthodes. Un suivi des prix des fruits de cueillette commercialisés en Guinée et une analyse approfondie de leur production et de leur utilisation ont été réalisés de mars 1998 à février 2000 sur deux mêmes marchés guinéens. Pour cela, les informations requises (prix, période, conditionnement, circuit de commercialisation) ont été collectées par enquêtes effectuées tous les quinze jours et consignées sur une fiche spécifique. Résultats. L'étude a mis en évidence d'éventuels problèmes d'approvisionnement des marchés, liés à leur site. Parmi les espèces identifiées, douze se sont révélées plus importantes et ont fait l'objet d'analyses. Leurs zones de production ont été définies. La majorité des fruits commercialisés sur les marchés enquêtés mûrissent entre mars et juin ; ils représentent une source de revenus pour une bonne part de la population. Les techniques de conservation, de commercialisation, de transformation et de consommation ont été répertoriées. Conclusion. L'enquête effectuée conduit à encourager la protection des fruitiers de cueillette et à conseiller leur introduction progressive dans les vergers familiaux. Les programmes de recherches nationaux cherchent à intervenir également dans la transformation et la conservation des produits issus de cueillettes, qui posent de grosses difficultés aux producteurs.

Guinée / espèce endémique / plante fruitière / cueillette / localisation des productions / prix de marché / utilisation 


\section{Introduction}

La République de Guinée est un pays côtier d'Afrique de l'Ouest, largement ouvert sur l'océan Atlantique [1]. Le pays jouit d'un climat de type tropical. Son relief est très accidenté, marqué par des plaines (alluviales, littorales et sédimentaires), des plateaux et des montagnes (chaîne de la dorsale guinéenne et massifs montagneux du Fouta Djallon et d'une partie de la Basse Guinée). Ce climat est caractérisé par de fortes précipitations favorisant un réseau hydrographique très important, à l'origine du surnom de "château d'eau de l'Afrique de l'Ouest " qui a pu être donné à la Guinée [2]. L'ensemble de ces éléments géographiques a permis d'identifier quatre régions naturelles au sein du pays : la Guinée maritime ou Basse Guinée, la Moyenne Guinée ou Fouta Djallon, la Haute Guinée et la Guinée forestière. Chacune de ces régions dispose d'une végétation spécifique et d'espèces fruitières dites "sauvages " adaptées à ces zones naturelles ; ces espèces fruitières poussent et se développent de façon spontanée dans les brousses guinéennes et produisent à diverses périodes de l'année. Si leurs fruits constituent l'alimentation usuelle de certains animaux de brousse (singes, oiseaux, etc.), ils sont progressivement entrés dans la consommation humaine. Si, anciennement "dans leur grande majorité, ces espèces fruitières ne sont consommées que par les populations qui les récoltent " [3], plusieurs d'entre elles sont actuellement commercialisées sur les marchés guinéens.

Compte tenu de l'importance que revêt, sur le marché local et sous-régional, l'usage alimentaire et commercial des fruits produits par ces espèces particulières, un programme du centre de Recherche agronomique de Foulaya (CRAF) en Guinée, le programme recherche système $(\mathrm{R} / \mathrm{S})$, a jugé utile de faire, au travers d'enquêtes successives, une analyse plus approfondie de leur production.

Une étude complémentaire a permis de suivre, de mars 1998 à février 2000, les prix de vente au détail de ces produits de cueillette sur deux marchés importants de Guinée. Ces marchés ont été sélectionnés à partir de critères liés à des caractéristiques géographiques, à l'ancienneté de leur implantation, à leur importance et à la diversité de leur environnement social :

- le marché Madina, dans la commune de Matam, est le plus gros des marchés de Conakry ; il est localisé dans une zone à extension limitée en raison des constructions environnantes,

- le marché Yenguéma est situé dans la ville de Kindia à proximité des zones de cueillette.

Le suivi des prix a eu pour objectif :

- d'identifier les fruits ou produits spontanés de cueillette consommés et commercialisés sur les marchés en Guinée,

- de mettre au point une base d'informations fiables sur les zones et les périodes de production de ces fruits, sur leur circuit de commercialisation et sur la fluctuation des prix, ainsi que sur les techniques de conservation, de transformation et de consommation de ces produits,

- d'orienter les travaux de recherche des programmes fruitiers, du programme de conservation phytogénétique et de celui des technologies agroalimentaires en cherchant à déterminer les produits les plus rentables ou méritant d'être rentabilisés. À partir des résultats obtenus, plusieurs actions peuvent être envisagées : mise en culture de certaines de ces espèces, mise au point de techniques de conservation phytogénétique ou recherche de techniques de traitement ou de transformation agroalimentaires.

\section{Matériel et méthodes}

Les enquêtes effectuées en 1999 sur les mêmes marchés que celles menées en 1998 ont permis de vérifier, par comparaison, les données acquises à l'issue de cette première année. À partir de l'ensemble des résultats, il a été également possible d'évaluer comment le site géographique des marchés et leur éventuel problème d'approvisionnement pouvaient interférer avec leur activité. 
Chaque marché a été visité tous les quinze jours. À chaque visite, les fruits ou produits de cueillette présents sur les étals ont été recensés ainsi que leur type de présentation (tas, unité ou poids) et leur prix. Les poids ont été évalués à l'aide d'un peson de $10 \mathrm{~kg}$ et les prix ont été notés en face du poids sur une fiche d'enquête qui spécifiait en outre l'identification botanique du produit, ses noms communs et locaux, son type de présentation, l'unité de vente utilisée, sa zone de production et son époque de commercialisation.

Les données annuelles du prix de détail par kg, obtenues à l'issue des enquêtes sur les différents produits, ont été consignées dans des tableaux.

\section{Résultats}

Les données collectées ont permis d'acquérir des informations sur la nature, le volume, la répartition et les prix des produits issus d'espèces fruitières spontanées consommées et commercialisées en Guinée.

La gamme de ces produits et de leurs dérivés s'est révélée importante (tableau I). La plupart d'entre eux se consomment sous forme de fruits (baobab, tamarin, prune du Japon ou Parinari excelsa, etc.) ; d'autres, subissent d'abord des transformations (beurre de karité, huile de palme, etc.) (tableau II). L'ensemble de ces produits représente un complément alimentaire précieux pour beaucoup de populations rurales (farine de néré, par exemple) ou constitue un simple apport vitaminique.

Les fruits entrent à maturité vers la fin de la saison sèche et au début de la saison des pluies (tableau II). Leurs zones de production ont pu être identifiées (tableau II) et, pour quelques produits, leur fluctuation sur les marchés a été définie. Les prix correspondant à ces périodes ont pu être déterminés. La vente de ces produits s'est révélée une source de revenu importante pour un grand nombre d'individus.

Pour compléter ces premières informations, les techniques de conservation, de commercialisation, de transformation et de consommation de certains de ces produits ont été précisées. En fait, la conservation nécessite souvent des traitements importants permettant une consommation diversifiée et prolongée de ces produits ou sousproduits durant l'année (tableau II).

Dans les villages de la Haute Guinée, Parkia biglobosa (le néré) et Vitellaria paradoxa (le karité) sont des plantes importantes et protégées qu'il est interdit de couper jeunes ou en âge de production. Leur bois ne peut être utilisé que si l'arbre est vieux ou tombé accidentellement du fait, par exemple, des vents en saison des pluies. Malgré leur développement naturel, la cueillette de leurs fruits est régie par une réglementation bien respectée : tout arbre fruitier d'une parcelle privée appartient à son propriétaire et le prêt du terrain à un tiers ne lui donne pas automatiquement droit à l'exploitation des arbres présents sur la parcelle. Cependant, dans les zones non occupées, la cueillette est libre à la condition qu'elle obéisse au calendrier la réglementant.

La synthèse de l'ensemble des informations obtenues a permis d'élaborer une fiche descriptive pour chacune de douze espèces fruitières présentant un intérêt alimentaire ou économique pour la population guinéenne.

\subsection{Uvaria aethiopica Guil. et Pers.}

Uvaria aethiopica Guil. et Pers. [4] est une Annonacée qui a également été appelée Xylopia aethiopica (Duval) A. Rich. [3] ou Unona aethiopica (Duval) [4]. Elle produit du poivre noir (tableau I). L'arbre a un feuillage léger, une écorce noire, des feuilles alternes et des fruits minces (figure 1), de (2 à 3) $\mathrm{cm}$ de long et disposés en grappe. La capsule noire, très odorante, contient des graines noires et dures.

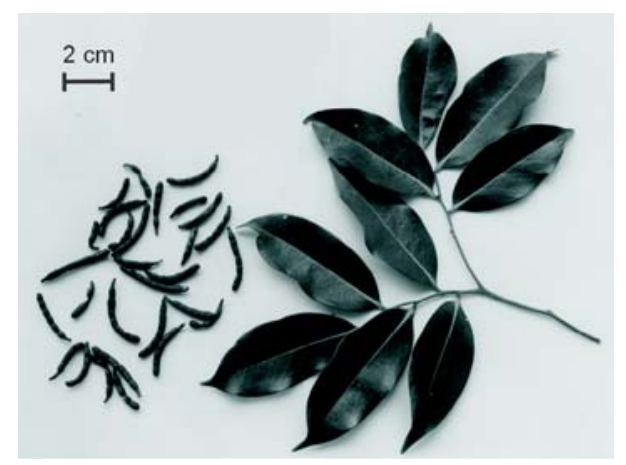

Figure 1.

Feuilles et fruits de Uvaria aethiopica ou « poivre de Guinée ». 


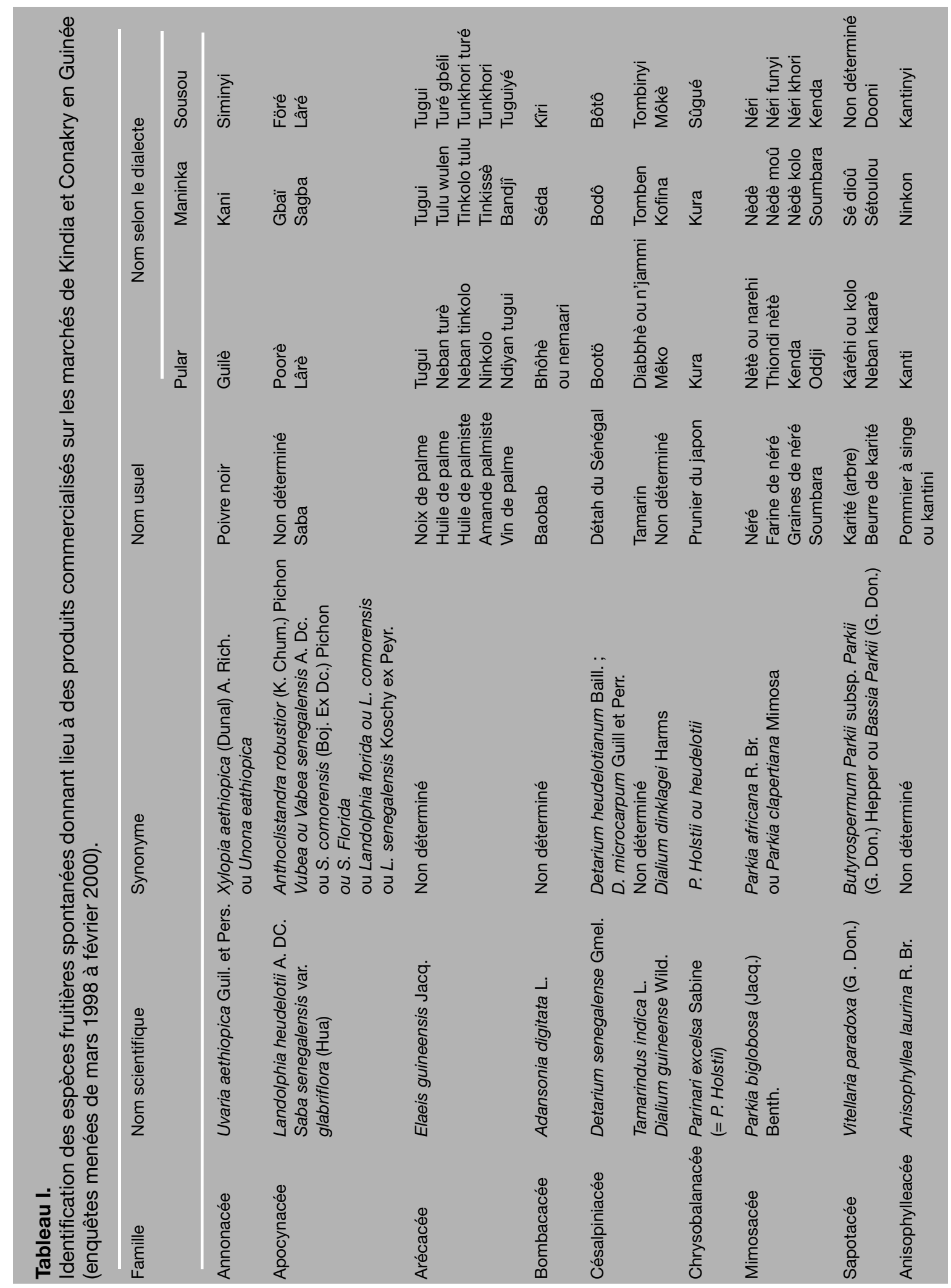

102 Fruits, vol. 58 (2) 


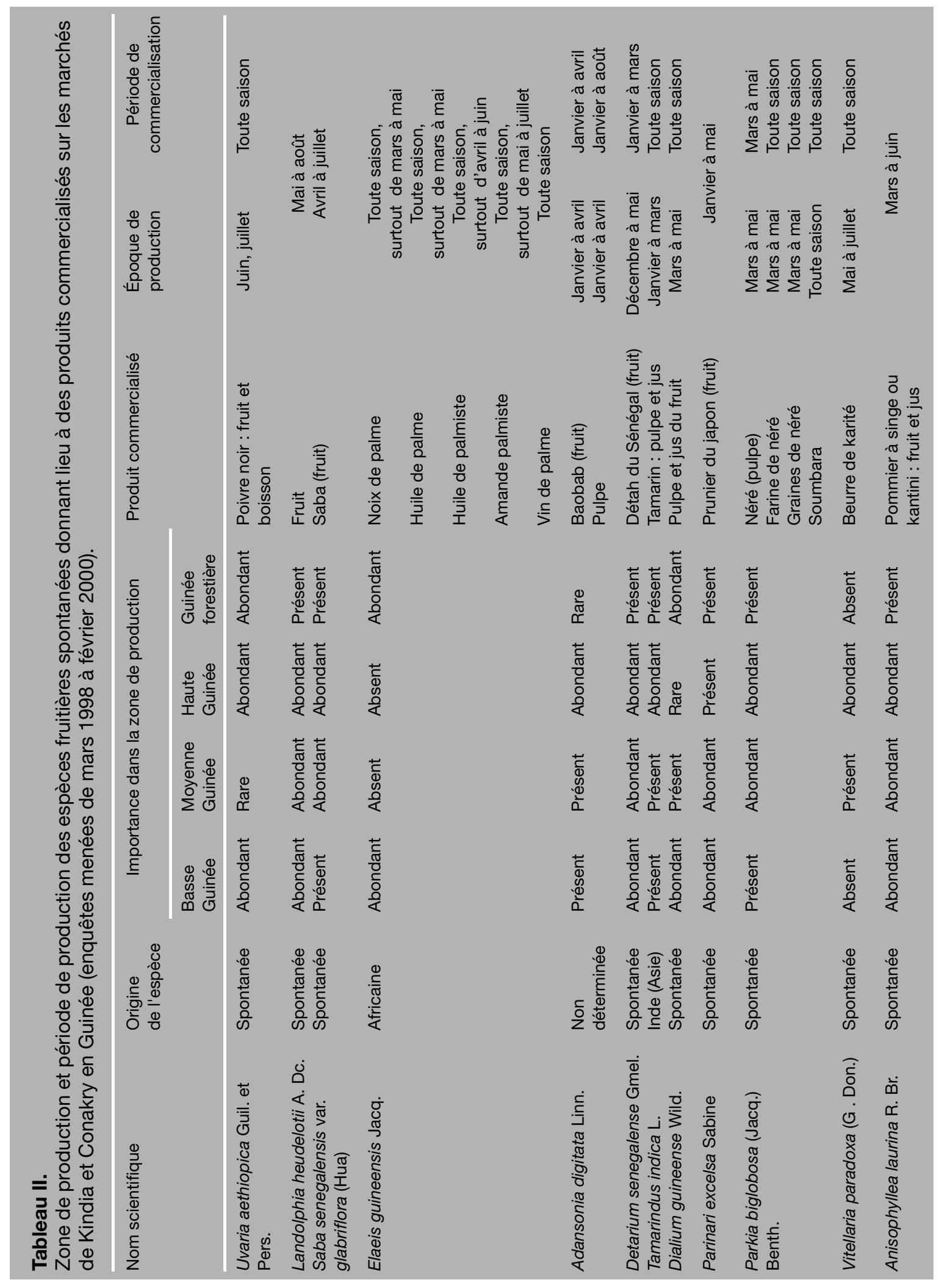




\section{Figure 2}

Périodes de production et évolution des prix, sur deux marchés, de produits issus de la production de quatre espèces fruitières spontanées en Guinée (mars 1998 à février 2000 ;

$1 €=1800$ francs guinéens).

\section{Figure 3.}

Fruits de Lando/phia heudelotii.

Le fruit est récolté entre juin et juillet dans tout le pays sauf dans la région de Moyenne Guinée où il est rare (tableau II).

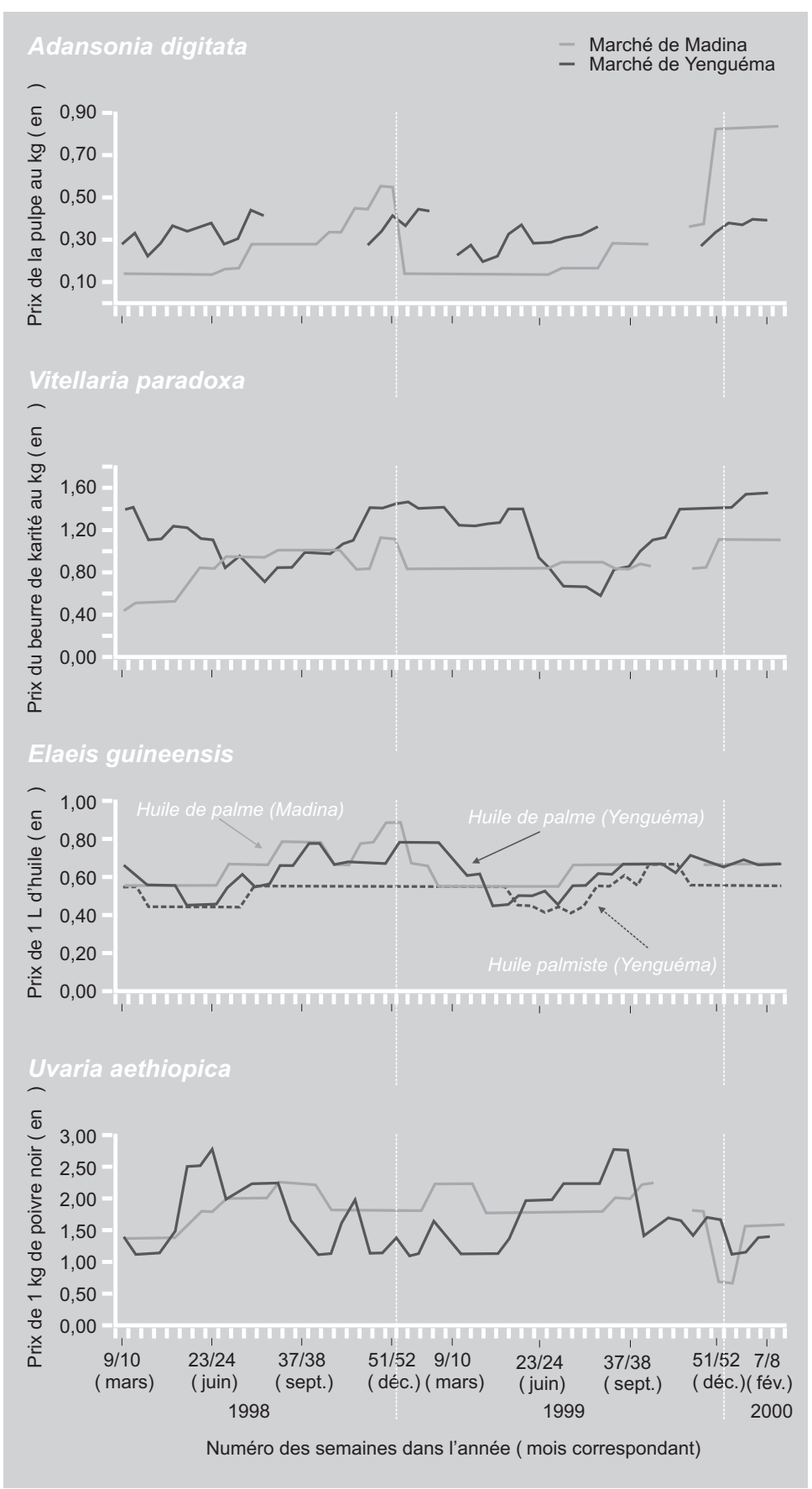

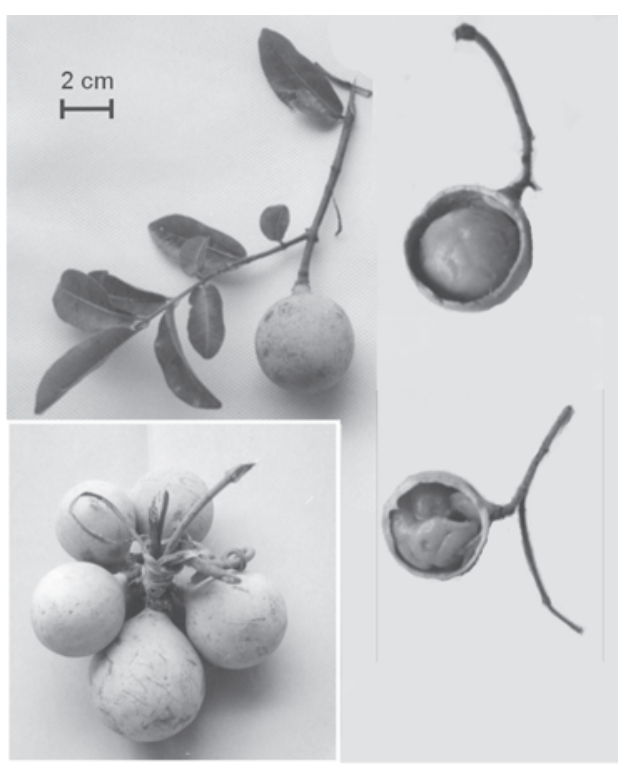

Pour la pharmacopée, les vertus du poivre de Guinée sont nombreuses. Il est utilisé pour le traitement des femmes en couche, comme épice dans certaines sauces et, en mélange avec du gingembre, il peut être consommé en tisane chaude (" café Touba ") et soigner alors le rhume et la toux.

Les fruits qui peuvent se conserver longtemps à l'état sec sont vendus en tas en toute saison. Les prix au $\mathrm{kg}$ varient autour de $(1,50$ à 2,00$) €^{1}$. Pendant la période d'étude, le marché de Yenguéma a connu des prix plus fluctuants que celui de Madina (Conakry) (figure 2).

\subsection{Landolphia heudelotii A. DC.}

Encore appelée Anthoclistandra robustior (K. Chum) Pichon [3], l'espèce L. heudelotii appartient à la famille des Apocynacées [4]. C'est un arbuste en lianes contenant du latex et formant le plus souvent un buisson. Ces lianes peuvent atteindre $15 \mathrm{~m}$ de haut et elles présentent des rameaux qui se vrillent. Les fruits sont des baies en grappes; leur peau est lisse et mince, jaune-orange à maturité (figure 3); la

\footnotetext{
${ }^{1} 1 €=1800$ francs guinéens.
} 
pulpe est blanche, sucrée et acidulée. Connue dans toute la Guinée, L. heudelotii est particulièrement abondante à Gbinkili près de Kindia.

Le fruit de L. heudelotii est utilisé dans la préparation de boissons rafraîchissantes et de bouillies dont il améliore le goût et rehausse la valeur nutritive.

La cueillette et la vente ont lieu au cours des mois de mai à août et les fruits sont vendus en tas plus ou moins gros sous forme de branchettes attachées les unes aux autres (figure 3$)$; le prix varie de $(0,10$ à 0,15$) € \cdot \mathrm{kg}^{-1}$ sur le marché de Yenguéma (Kindia) proche d'une zone de production et il est de l'ordre de $0,25 € \cdot \mathrm{kg}^{-1}$ sur le marché Madina à Conakry, qui doit prendre en compte des frais de transport routier (figure 4). Compte tenu de la démographie, la consommation à Conakry se révèle élevée.

\subsection{Saba senegalensis var. glabriflora (Hua) Pichon}

L'espèce Saba senegalensis appartient à la famille des Apocynacées. Dans la littérature, on lui trouve un certain nombre de synonymes [4-6] (tableau I). La plante est une liane à latex blanc qui pousse surtout au bord des rivières [4] et dans les zones de savanes arborées ; l'écorce est crevassée, écailleuse et de couleur grise. Cette liane forme un grand buisson, souvent entourant un arbre. Elle a des feuilles simples, opposées, ovales, obtuses au sommet ou arrondies à la base [4]. Le pétiole présente un long pédoncule.

Les fruits, ou sabas, sont des baies ovoïdes, de couleur jaune-orange à maturité contenant sept à dix noyaux recouverts d'une pulpe juteuse acidulée. La section du fruit montre une peau dure, épaisse et bosselée, qui recouvre une membrane fibreuse collée à la peau enveloppant l'ensemble des noyaux (figure 5). Cette membrane protège les noyaux et leur pulpe contre le latex blanc contenu dans l'enveloppe épaisse.

Les lianes peuvent servir de liens pour attacher les clôtures et les toitures des cases; le latex peut être utilisé comme colle à papier ; la pulpe du fruit, additionnée aux bouillies traditionnelles à base de néré (fruit de Parkia biglobosa), permet de rehausser le goût et la valeur nutritive de ces aliments; elle est aussi utilisée pour faire des boissons locales rafraîchissantes. Par ailleurs, les noyaux peuvent être simplement sucés par les
Figure 4.

Périodes de production et évolution des prix, sur deux marchés, des fruits de cueillette de quatre espèces fruitières spontanées en Guinée (mars 1998 à février $2000 ; 1 €=1800$ francs guinéens).

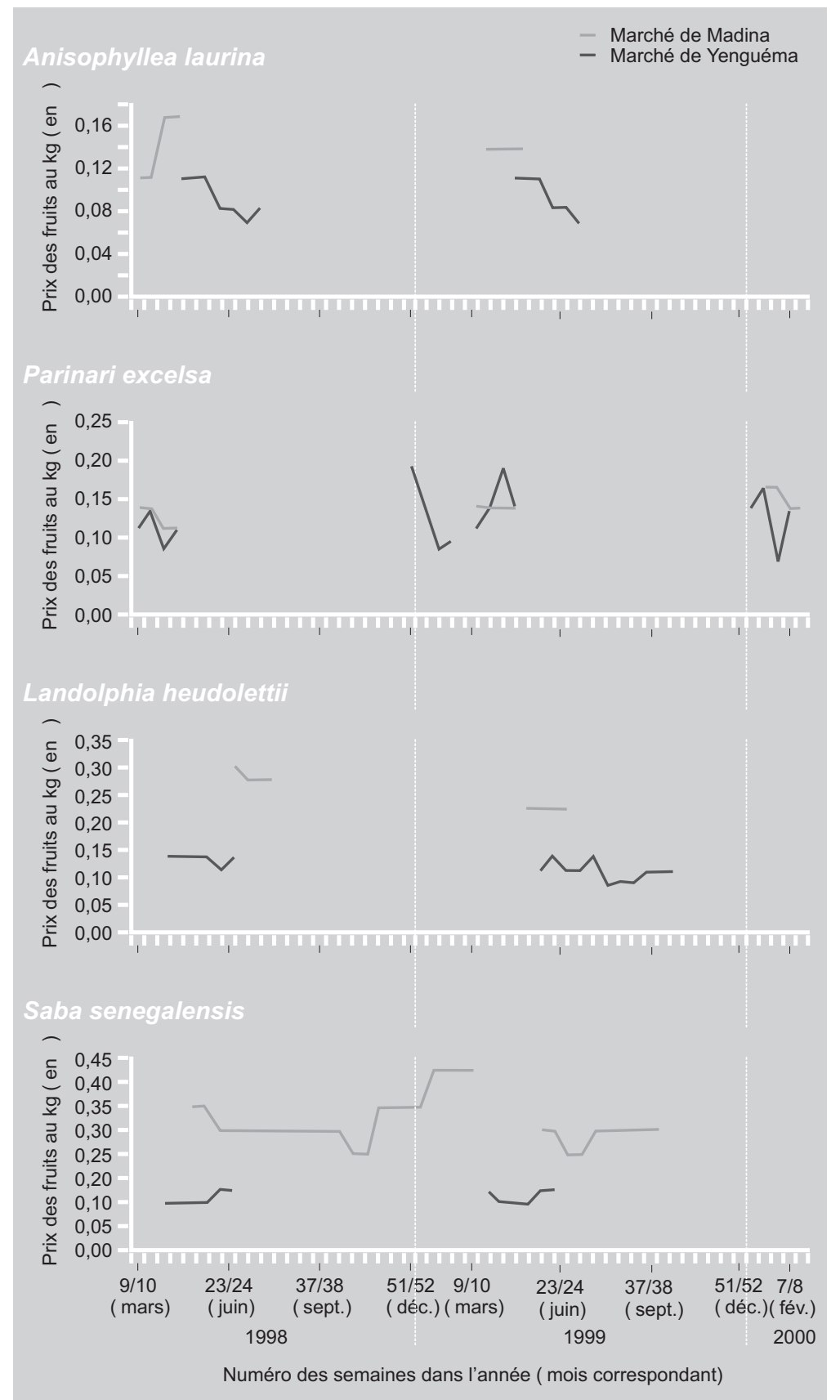


Figure 5.

Fruits de Saba senegalensis.

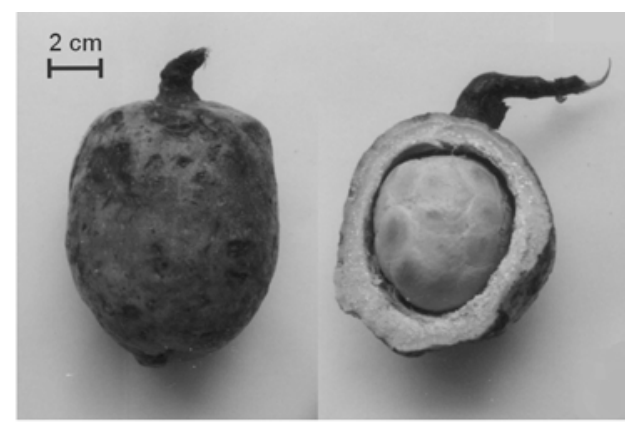

enfants. En Haute Guinée, ces noyaux entourés de leur pulpe sont séchés au soleil et utilisés pour remplacer le citron et le tamarin, rares durant les premiers mois de la saison des pluies.

De nos jours, le saba fait l'objet d'un commerce intense à Conakry où ses fruits sont acheminés dans des sacs ayant été utilisés précédemment pour le conditionnement de riz. Cependant, une grande partie de la production guinéenne est exportée vers le Sénégal par voie routière. Cette commercialisation sur de longs trajets est facilitée par la longue durée de conservation du fruit qui peut se maintenir en bon état pendant (8 à 10) jours s'il bénéficie de bonnes conditions de transport et de stockage. La production de $S$. senegalensis a lieu d'avril à juillet dans toute la Guinée, mais elle est particulièrement abondante en Moyenne et Haute Guinée. Le fruit est
Carte des zones de production de Elaeis guineensis en Guinée et flux commerciaux de l'huile de palme produit par le pays. vendu aux environs de $0,10 \quad € \cdot \mathrm{kg}^{-1}$ à Kindia (marché de Yenguéma) et $0,30 € \cdot \mathrm{kg}^{-1}$ à Conakry (marché de Madina) (figure 4).

\subsection{Elaeis guineensis Jacq.}

Elaeis guineensis ou palmier à huile appartient à la famille des Arécacées [3]. Cet arbre peut atteindre de (20 à 30) $\mathrm{m}$ de haut et $40 \mathrm{~cm}$ de diamètre. Ses feuilles sont grandes, groupées en bouquet au sommet de l'arbre. Il est cultivé pour ses fruits qui sont des drupes groupées en régimes [5]. En Guinée, le palmier à huile est exploité en Basse Guinée et en Guinée forestière (tableau II). Les principaux produits issus du palmier à huile et commercialisés sur les marchés enquêtés sont les noix de palme, l'huile de palme, l'huile de palmiste, les amandes de palmiste et le vin de palme. Cependant, les amandes de palmistes ne sont vendues que sur le marché Madina à Conakry.

- Sur les marchés de Kindia et de Conakry, les noix de palme présentes toute l'année sont commercialisées en tas vendus $0,013 €$ l'un. Elles rentrent dans la préparation de différentes sauces. Le kg coûte un peu plus de $0,15 €$ en mai et environ $0,55 €$ en novembre.

- Les huiles de palme et de palmiste sont vendues en toute saison sur les marchés étudiés. L'huile de palme est obtenue à partir de la pulpe des noix de palme tandis que l'huile de palmiste est obtenue à partir du traitement des amandes. Les zones de production de ces deux huiles sont la Basse Guinée et la Guinée forestière (figure O). Le litre d'huile de palme coûte entre $0,45 €$ en avril et $0,80 €$ en novembre, tandis que le litre d'huile de palmiste varie de $0,45 €$ à $0,60 €$ selon la saison (figure 2).

La technologie d'extraction de l'huile de palme varie d'une communauté à l'autre. En Guinée, deux procédés principaux ont été observés: le procédé par cuisson (figure 7) est très utilisé en Basse Guinée et dans une partie de la Guinée forestière et le procédé par fermentation (figure 8) est surtout connu en Guinée forestière. De l'avis de certains revendeurs interrogés sur les marchés, l'huile obtenue à partir de la 
méthode d'extraction par cuisson serait de meilleure qualité que celle obtenue par fermentation.

L'extraction de l'huile de palmiste requière une technologie particulière (figure 9). Elle est réalisée par les femmes.

\section{Figure 7.}

Technologie d'extraction de l'huile de palme par la méthode par cuisson, telle qu'elle est pratiquée en Guinée.

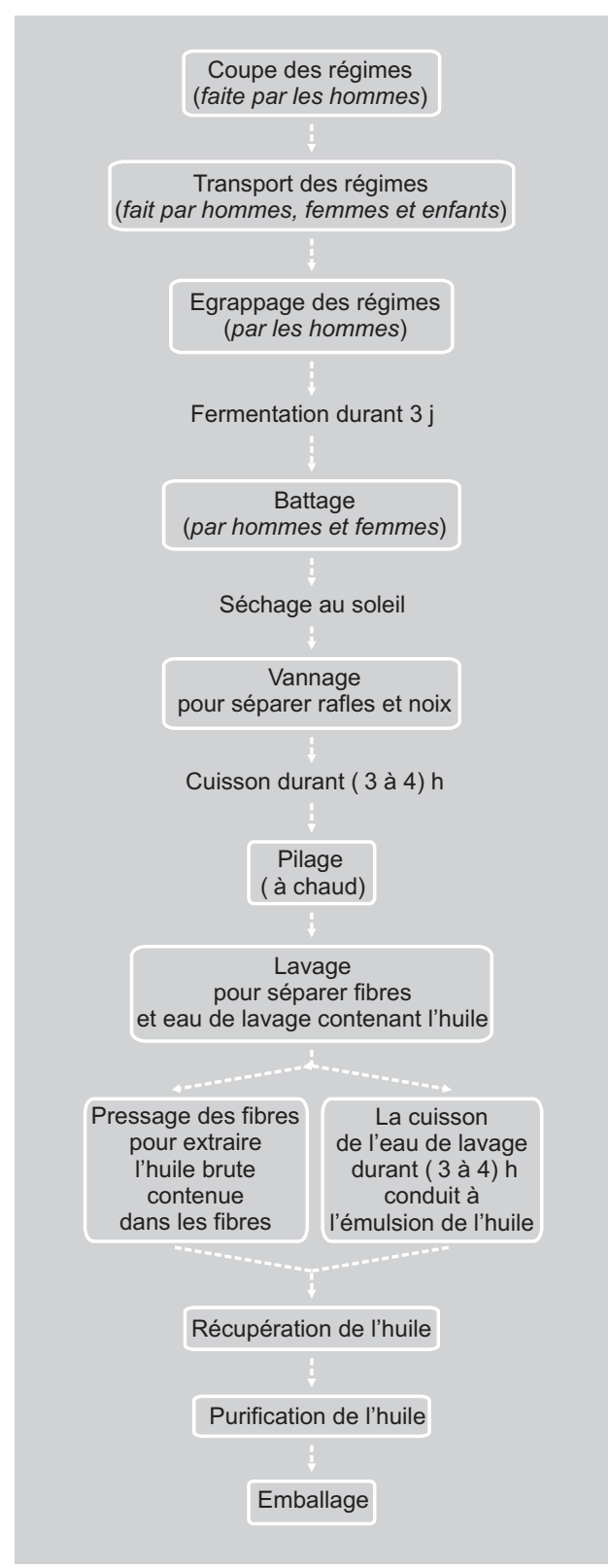

En Guinée, l'huile de palme et l'huile de palmiste sont utilisées dans l'alimentation et dans l'industrie, essentiellement pour la préparation du savon.

- Le vin de palme, obtenu à partir de l'extraction et de la fermentation de la sève du palmier à huile, est utilisé comme

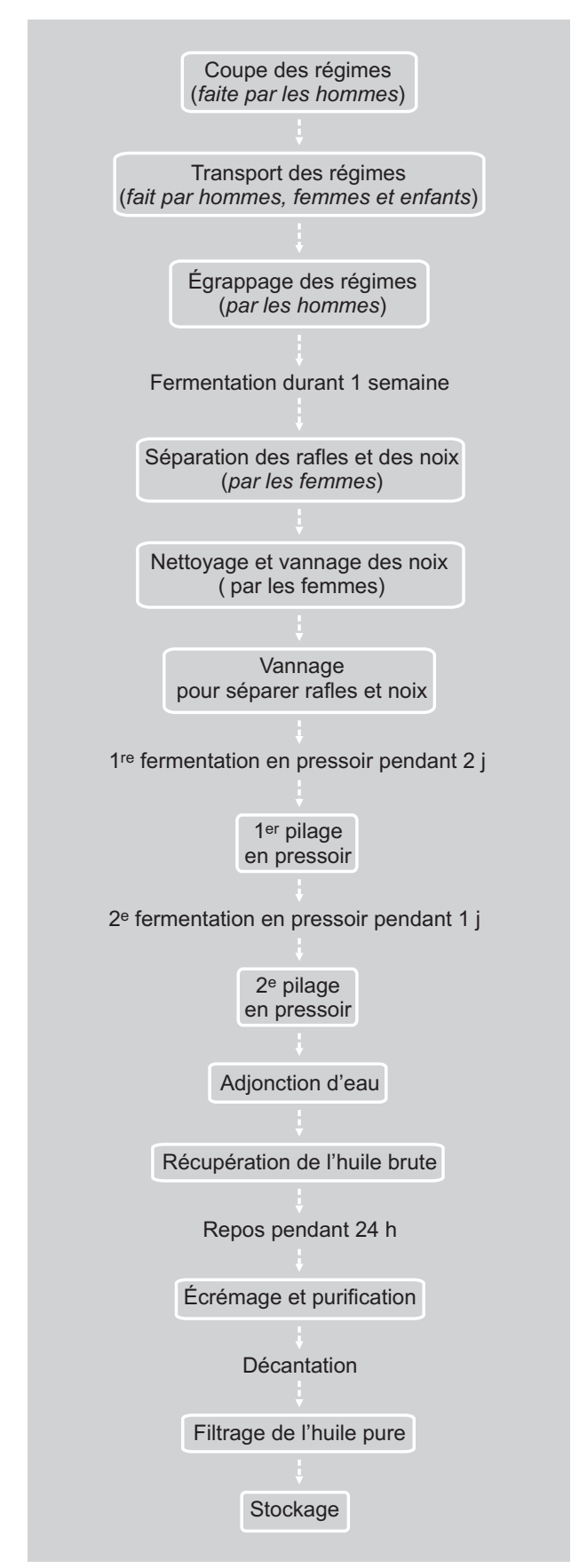

Figure 8.

Technologie d'extraction de I'huile de palme par la méthode par fermentation, telle qu'elle est pratiquée en Guinée. 


\section{Figure 9.}

Technologie d'extraction de I'huile de palmiste utilisée par les femmes en Guinée.
Figure 10.

Baobab (Adansonia digitata).

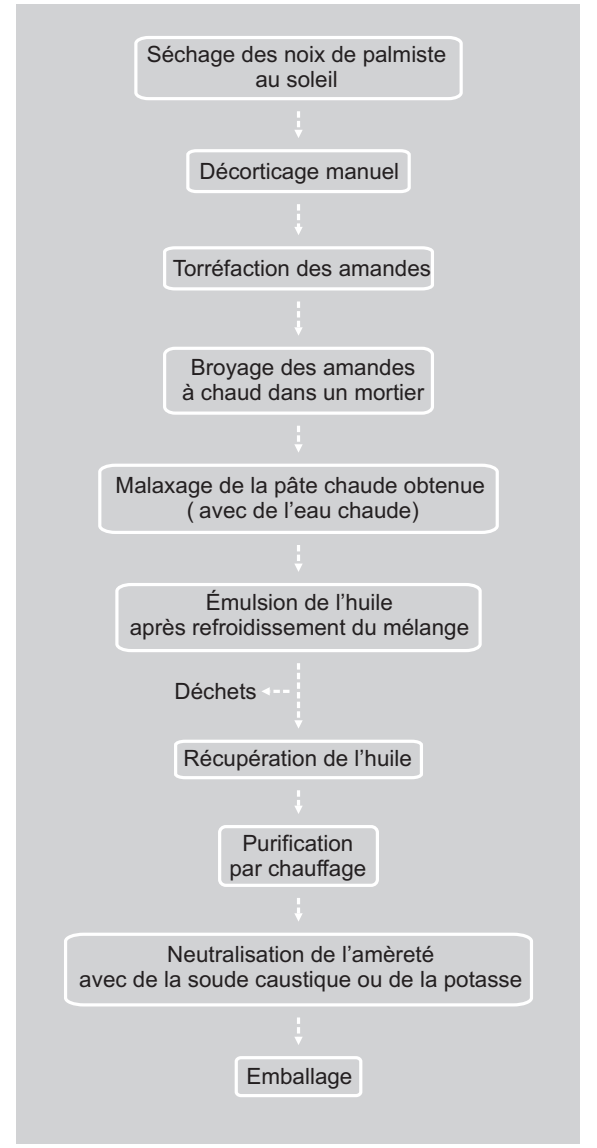

boisson. Il est efficace pour le traitement de la rougeole par absorption par le malade du liquide nouvellement récolté ou en lui enduisant le corps. D'après les vendeuses interrogées sur les marchés étudiés, le prix du litre de vin de palme varierait entre 0,10 et $0,14 €$.

\subsection{Adansonia digitata L.}

Adansonia digitata, connu sous le nom de baobab ou pain de singe, appartient à la

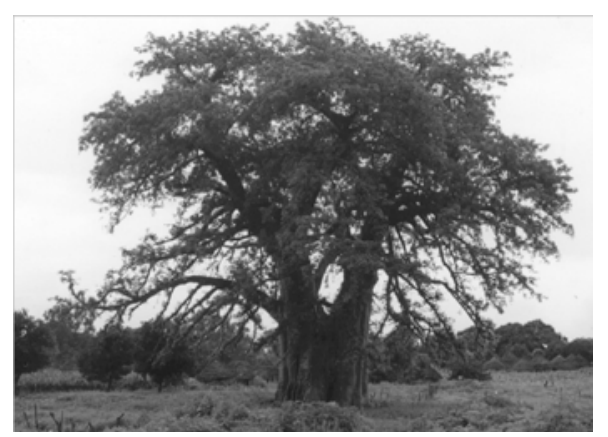

famille des Bombacacées [3]. C'est un grand arbre de (20 à 25) $\mathrm{m}$ de haut; son tronc, parfois creux lorsque l'arbre est très âgé, peut atteindre plus de $5 \mathrm{~m}$ de diamètre [5] (figure 10). Les feuilles sont alternes, composées et digitées à cinq folioles. Il donne des fruits en capsules appelés aussi " pains de singe "qui sont accrochés à l'arbre par un long pédoncule (figure 11). L'enveloppe pelliculeuse et dure, recouverte d'une poudre piquante, contient une pulpe farineuse blanchâtre, parfois légèrement colorée en rouge, dans laquelle sont noyées plusieurs graines dures et brunes et de nombreuses fibres. En Guinée, le baobab produit entre janvier et mars et surtout dans les savanes sahéliennes de Haute Guinée et dans la préfecture de Mali en Moyenne Guinée.

Le fruit est riche en acide ascorbique, en thiamine, en potassium, en glucose ainsi qu'en plusieurs autres vitamines [6]. La pulpe est consommée pure ou en association avec d'autres produits. Dissoute dans l'eau froide, c'est une boisson rafraîchissante. À Dinguiraye, une préfecture de la Haute Guinée, une recette traditionnelle permet de préparer le "souloukoubada" (ou "gourde de l'hyène" en dialecte Maninka) : après avoir ouvert un trou à l'extrémité du fruit fixée au pédoncule, on

Figure 11.

Feuilles et fruit d'Adansonia digitata encore appelé « pain de singe ».

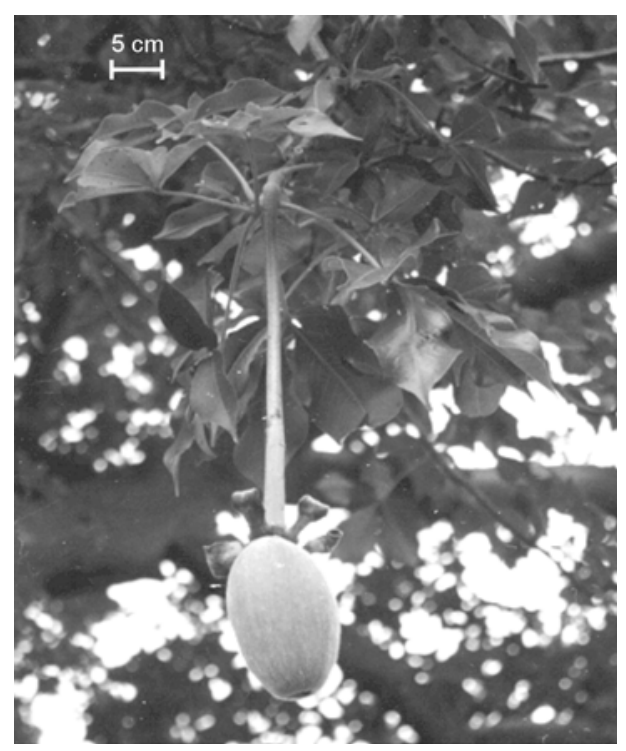


verse de l'eau dans la capsule jusqu'à ce que la pulpe soit bien humectée; de la pâte d'arachide, du sucre ou du miel sont alors ajoutés à cette pulpe humide et mélangés à l'aide d'une baguette jusqu'à obtenir une pâte semi-liquide consommée telle que. Par ailleurs, en Guinée, la pulpe du baobab, associée à de la farine de néré (issue du fruit de Parka biglobosa) permet la préparation d'un bonbon glacé (le "kîri bonbon ") qui est le plus apprécié de tous les bonbons glacés (jus vendus sous forme de glaçons) vendus sur les marchés guinéens.

Les feuilles du baobab sont très riches en calcium et en fer [5]. Séchées et réduites en poudre, elles sont utilisées dans la préparation de sauces ou servent à lier les grains du couscous.

Le fruit existe sur les marchés durant la période de récolte de janvier à avril, tandis que la pulpe séchée est vendue sur les marchés presque toute l'année. Le prix du fruit varie entre $(0,06$ et 0,17$) €$ selon la taille du fruit. La pulpe séchée quant à elle coûte entre $(0,14$ et 0,83$) € \cdot \mathrm{kg}^{-1}$ à Conakry en fonction des saisons et entre $(0,22$ et $0,44) € \cdot \mathrm{kg}^{-1}$ à Kindia (figure 2).

\subsection{Detarium senegalense Gmelin.}

Appelé aussi Detarium beudelotianum Baill. [4] ou Detarium microcarpum Guill. et Perr. [5], D. senegalense Gmel., le détah du Sénégal ou "bootö", appartient à la famille des Césalpiniacées. Il y a deux sortes de Detarium qui se ressemblent beaucoup : le Detarium comestible (Detarium senegalense) et le Detarium amer et toxique. L'espèce comestible comporte elle-même deux variétés: l'une dont les fruits plus gros sont vendus sur les marchés et l'autre dont les fruits plus petits sont peu connus des consommateurs (figure 12). Cette deuxième variété comestible serait, selon M. Camara botaniste au laboratoire de protection des végétaux de Foulaya (Guinée), le Detarium guineensis. Les fruits du Detarium toxique mûrissent plus tard que ceux des variétés comestibles et, même mûrs et tombés par terre, ils restent intacts, non consommés par les animaux. Les arbres des deux espèces sont présents et bien identifiés sur la parcelle expérimentale du centre de recherche agronomique de Foulaya (Kindia, Guinée).

L'arbre de (15 à 30) $\mathrm{m}$ de haut [4] est présent dans les quatre régions naturelles de la Guinée. Les feuilles sont à folioles alternes et les fruits sont globuleux ; ils contiennent un gros noyau recouvert d'une pulpe farineuse légèrement acidulée, avec beaucoup de fibres (figure 12). Ces fruits sont récoltés entre janvier et mars et on les trouve parfois sur les marchés à partir de novembre. Ils sont vendus entre $(0,01$ et 0,03$) €$ pièce ; à Conakry, ils sont commercialisés entre $(0,08$ et 0,28$) € \cdot \mathrm{kg}^{-1}$ et à Kindia, où l'espèce n'est pas beaucoup exploitée, les prix varient entre $(0,22$ et 0,36$) € \cdot \mathrm{kg}^{-1}$ (figure 13). Les autres zones de récolte alimentent directement le marché de Conakry.

La pulpe est consommée directement quand le fruit est mûr ; c'est la seule forme de consommation connue actuellement. Les graines brûlées chassent les moustiques. La gomme-résine extraite des écorces, très odorante, est utilisée pour la fumigation des cases et des vêtements.

\subsection{Tamarindus indica $\mathrm{L}$.}

Tamarindus indica L., ou tamarinier, appartient à la famille des Césalpiniacées. L'arbre atteint une quinzaine de mètres de hauteur et $80 \mathrm{~cm}$ de diamètre [3]. Il peut pousser jusqu'à $1500 \mathrm{~m}$ d'altitude [5]. Les climats semi-arides ( $400 \mathrm{~mm}$ de pluie) lui conviennent aisément. L'écorce est grise, crevassée et écailleuse. Les feuilles sont petites, persistantes et alternes. Les fruits sont des gousses de 5 à $15 \mathrm{~cm}$ de long, subcylindriques et aplaties, courbées et en grappes sur l'arbre. Ces gousses sont brunes à maturité (figure 14) ; elles contiennent des graines entourées d'une pulpe rouge-brun, collante et très acidulée. En Guinée, le tamarinier est surtout abondant en Haute Guinée mais on le trouve également dans les trois autres régions. Il produit de janvier à mars. Un arbre en pleine production peut donner entre (150 à 200) kg de fruits par an [6]
Figure 12

Fruits entiers et décortiqués

de la variété comestible

de Detarium senegalense.

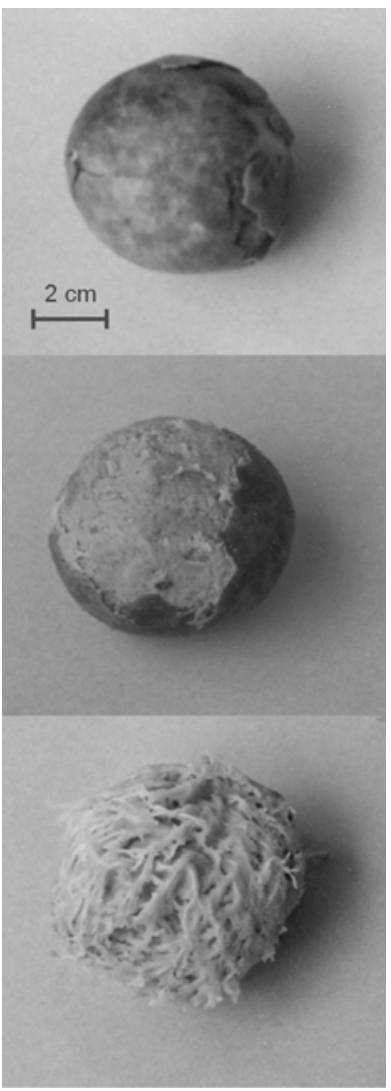




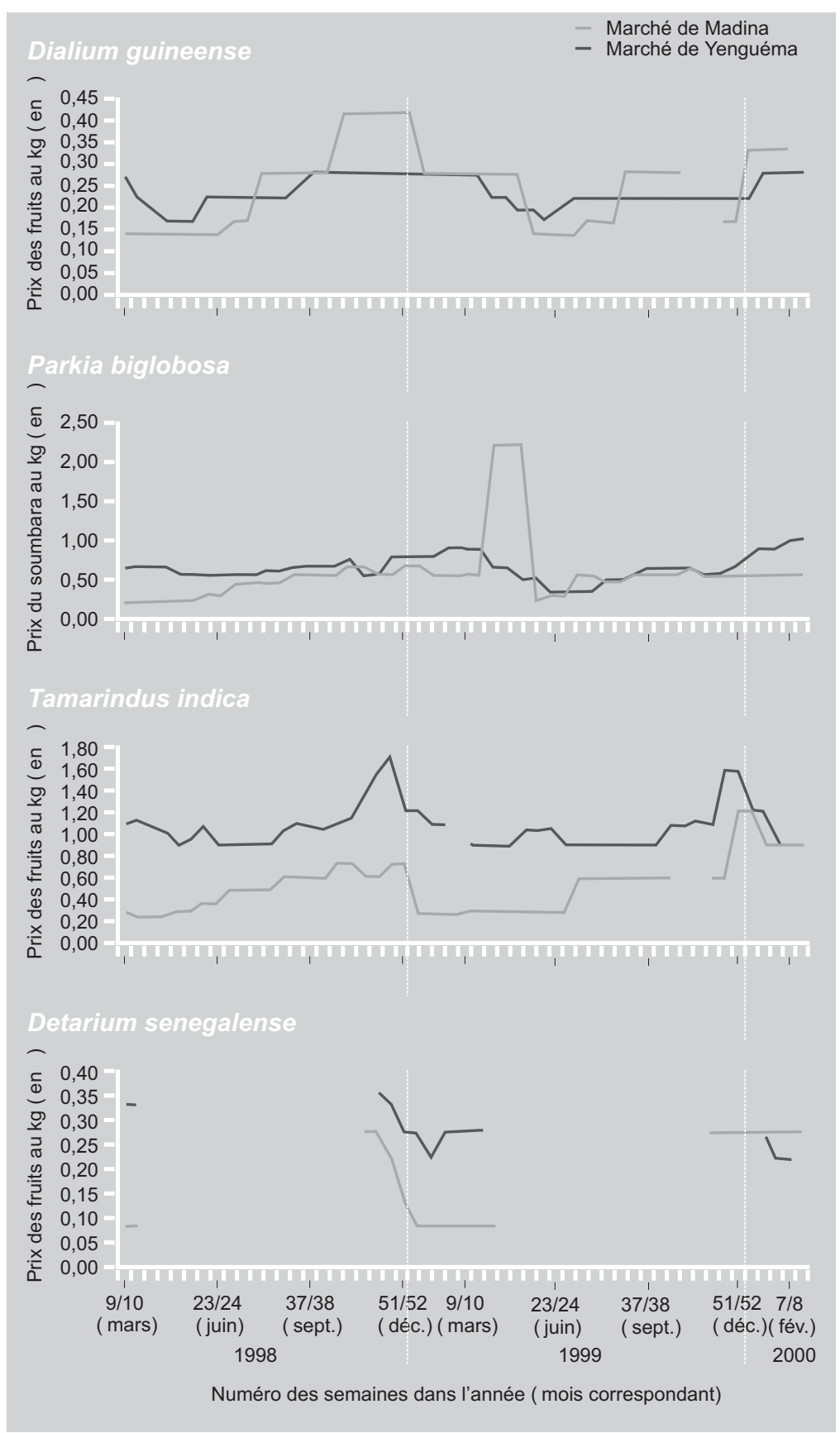

\section{Figure 13.}

Périodes de production et évolution des prix, sur deux marchés, de produits issus de la production de quatre espèces fruitières spontanées en Guinée (mars 1998 à février $2000 ; 1 €=1800$ francs guinéens). lier ce problème, cette pulpe peut être traitée par la vapeur et être alors conservée dans un récipient propre hermétiquement fermé.

La pulpe est consommée dans des bouillies et des boissons traditionnelles pour améliorer le goût ou pour apporter un complément nutritionnel. En mélange avec du gingembre, elle entre aussi dans la préparation de boissons glacées. Elle est utilisée dans le traitement de la gale et de la rougeole.

En écrasant l'enveloppe sèche du fruit mûr, on peut récupérer la pulpe qui, commercialisée en boulettes, peut être trouvée sur les marchés en toute saison. Les prix varient de $(0,25$ à 0,66$) € \cdot \mathrm{kg}^{-1}$ à Conakry et de $(0,83$ à 1,56$) € \cdot \mathrm{kg}^{-1}$ à Kindia (figure 13).

\subsection{Dialium guineense Wild.}

Appelé aussi Dialium dinklagei Harms [3], Dialium guineense Wild ou tamarinier noir [5] appartient à la famille des Césalpiniacées (tableau I).

Cet arbre, qui pousse le long des cours d'eau, est très abondant autour de Kindia. Il produit de petits fruits noirs, légèrement plus gros que la graine de café, disposés en grappes ; la chair est rougeâtre, farineuse, légèrement acidulée et contient de petites graines noires (figure 15). L'écorce, les feuilles et les racines bouillies sont utilisées en pharmacopée.

Le fruit est commercialisé sous diverses formes: les fruits non décortiqués sont vendus en période de récolte, de mars à mai, soit en branches portant les fruits en grappes, soit en tas ; les fruits décortiqués peuvent être consommés tel quel ou conditionnés en pots ou en sachets plastiques; macérés dans le l'eau froide, ils permettent d'obtenir une boisson rafraîchissante ou des "bonbons glacés" (ou "mookè bonbon") présentés sous sachets plastiques. Ils sont alors trouvés toute l'année.

Le prix des fruits varie selon les saisons entre $(0,13$ et 0,42$) € \cdot \mathrm{kg}^{-1}$ à Conakry et entre $(0,16$ et 0,28$) € \cdot \mathrm{kg}^{-1}$ à Kindia (figure 13). 


\subsection{Parinari excelsa Sabine (= P. Holstii)}

De la famille des Chrysobalanacées, le Parinari excelsa Sabine [3] ou " kura " dans le dialecte pular est appelé aussi prunier du Japon [4]. C'est un grand arbre de $50 \mathrm{~m}$ de haut et $1,50 \mathrm{~m}$ de diamètre. Il pousse dans toutes les zones tropicales d'Afrique. Les feuilles sont persistantes, alternes et simples.

Le kura produit un fruit en drupe ronde, à peau rousse contenant un gros noyau entouré par une pulpe charnue de couleur jaunâtre, odorante. Il est produit partout en Guinée (tableau II).

Les fruits parviennent à maturité entre janvier et mai. Ils sont alors directement consommés. Ils peuvent cependant être aussi vendus sur les marchés par les enfants ou les femmes, soit à l'unité soit en tas (cinq à dix fruits pour $0,013 €$ ). Le prix au $\mathrm{kg}$ varie entre $(0,11$ et 0,14$) € \cdot \mathrm{kg}^{-1}$; il atteint rarement les $0,19 € \cdot \mathrm{kg}^{-1}$ (figure 4 ). Si en Moyenne Guinée, il semble pouvoir être consommé tel quel ou pilé pour en obtenir une boisson fraîche qui serait une sorte de bière légère [7], cette forme de préparation du jus n'est pas développée.

\subsection{Parkia biglobosa (Jacq.) Benth.}

Encore appelé Parkia africana R. Br. ex G. Don. [8], P. biglobosa, localement nommé le " néré ", est un arbre qui peut atteindre $20 \mathrm{~m}$ de haut et $1,6 \mathrm{~m}$ de diamètre [8]. Il appartient à la famille des Mimosacées et pousse dans les savanes tropicales d'Afrique. Il est protégé dans les jachères. Les feuilles caduques sont alternes et bipennées. Les fruits sont de longues gousses, aplaties, de couleur brun foncé contenant de nombreuses graines noires entourées d'une pulpe poudreuse, jauneverdâtre et riche en vitamine C [3]. Un $\mathrm{kg}$ de gousses de néré compte de 4000 à 5000 graines. La production annuelle d'un arbre varie entre (25 et 100) kg de gousses. Une gousse renferme $20 \%$ de graine et $40 \%$ de pulpe.

Le néré est récolté en saison sèche de mars à mai. L'arbre pousse et se développe

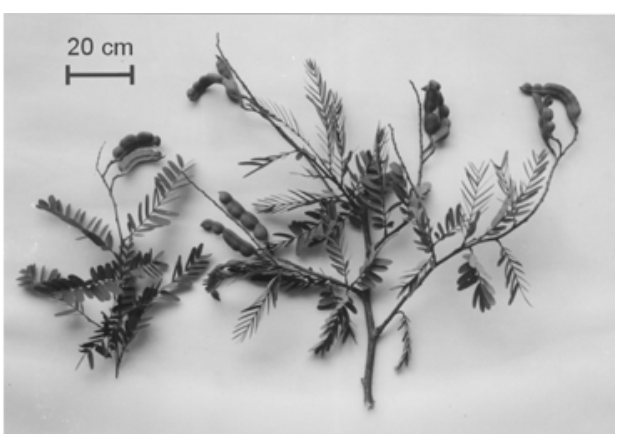

Figure 14.

Feuilles et fruits de Tamarindus indica.

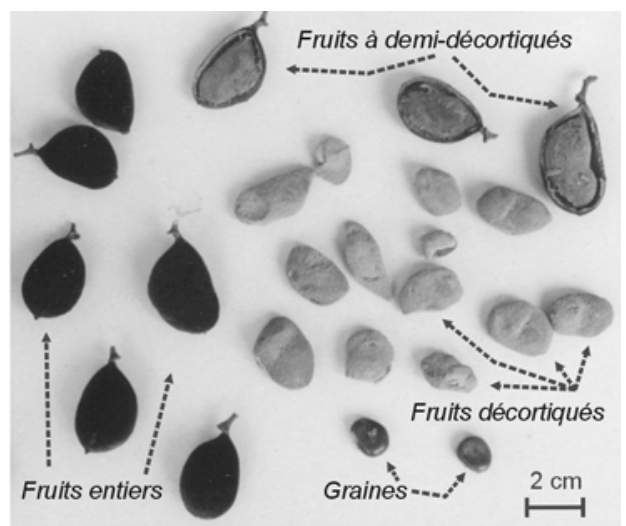

Figure 15.

Fruits et graines de Dialium senegalense.

dans toutes les savanes guinéennes. Particulièrement abondant en Haute et Moyenne Guinée, il n'est fréquent en Basse Guinée qu'à Kindia, Télimélé et Friah, et en Guinée forestière, à Kissidougou, Guékédou et une partie de Beyla (figure 10).

L'arbre est très utile car les écorces sont utilisées en pharmacopée pour soigner diverses maladies. La cendre du bois renferme de la potasse utilisée pour la préparation du savon noir. La pulpe des fruits est comestible et leurs épluchures sont utilisées pour la pêche artisanale dans les petits cours d'eau, en saison sèche: ces épluchures jetées à l'eau s'y décomposent et entraînent l'engourdissement puis la mort des poissons, mais elles ne sont pas toxiques pour l'homme. Cette pratique, qui est nocive pour tous les poissons quels que soient leur âge et leur espèce, serait à proscrire.

Les gousses du néré donnent lieu à divers produits commercialisés : pulpe, farine, graines et soumbara qui est un condiment noirâtre entrant dans la 


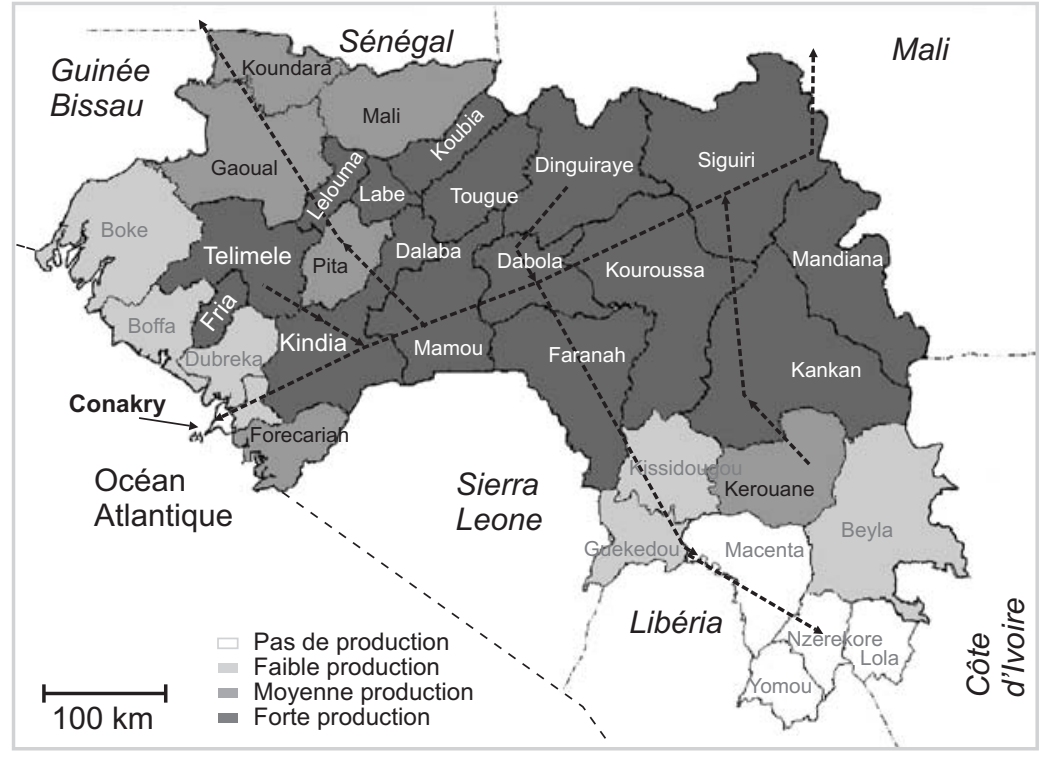

Figure 16.

Carte des zones de production de Parkia biglobosa en Guinée et flux commerciaux de son fruit, le néré.

\section{Figure 17}

Technologie d'extraction des graines des gousses de Parkia biglobosa ou " nérés ", et obtention de farine (Guinée). composition de diverses sauces. Parmi ces quatre formes trouvées sur les marchés, les plus courantes sont le soumbara, produit le plus cher, puis la farine de néré.

La pulpe, qui contient les graines, est obtenue après épluchage. Elle est vendue en sachets plastiques, à $0,03-0,05 €$ le sachet, pendant la principale saison de récolte de néré, de mars à mai (tableau II). Cependant ce type de commercialisation n'est pas économique car, après consommation de la pulpe, les acheteurs rejettent les graines qui auraient pu servir à un autre usage.

La farine séchée, qui est obtenue de la pulpe après extraction des graines (figure 17), est également vendue en sachets plastiques. Plus les gousses sont mûres au moment de la cueillette, meilleure est la farine qui doit être alors très jaune et sucrée. Ce produit est sensible aux attaques d'insectes, mais traité à la vapeur d'eau, il peut être conservé un certain temps en récipient propre hermétiquement fermé. La farine de néré peut être consommée telle quelle, crue, mais, sous cette forme, elle est collante et donc désagréable à manger. Elle est donc plus souvent consommée sous forme de pâte, en mélange avec des produits acides comme le tamarin, le saba (fruit de Saba senegalensis), le citron ou le lait de vache caillé.

Dans certains villages de la préfecture de Kankan (Haute Guinée), la farine de néré est usuellement consommée en saison des pluies pendant les travaux en champs. Elle est alors mélangée à du couscous de manioc et à du lait caillé. Outre son rôle alimentaire, elle sert dans ce cas à donner un goût sucré au mélange.

En Basse Guinée, la farine de néré est utilisée comme ingrédient dans la préparation de boissons glacées vendues sur les marchés en saison. Actuellement le programme de technologie agroalimentaire du Centre de recherche agronomique de Foulaya (Kindia, Guinée) est en train d'expérimenter la préparation de biscuits à base de farine de néré.

La farine est vendue en sachets plastiques entre $(0,14$ et 0,44$) € \cdot \mathrm{kg}^{-1}$. 
Les graines ne sont pas vendues au détail sur les marchés de Conakry. En revanche, elles sont trouvées sur les marchés hebdomadaires de collecte en milieu rural. Si les femmes préparent du "soumbara " avec les graines de néré, les hommes en font du commerce à destination des pays voisins (figure 10) où ces graines subissent une transformation industrielle aboutissant à production de cubes pour sauces.

Le soumbara (ou soumbala), condiment préparé artisanalement, est riche en calcium, en phosphore et en vitamine A [5]. Obtenu à partir de la cuisson et de la fermentation des graines de néré (figure 18), il est présent sur les marchés en toute saison. Il est produit partout en Guinée, mais surtout à Ballet dans la préfecture de Mamou (Moyenne Guinée), à Kindia (Basse Guinée) et dans toute la Haute Guinée.

Les prix du soumbara varient selon le conditionnement et la saison. Il peut être ainsi commercialisé en sachets, en pots, en mottes ou en tas selon les lieux. À Conakry, le soumbara est vendu de $(0,22$ à $0,67) € \cdot \mathrm{kg}^{-1}$ et, à Kindia, de $(0,33$ à 0,89) $€ \cdot \mathrm{kg}^{-1}$ (figure 13).

\subsection{Vitellaria paradoxa Gaertn.}

Appelé aussi Butyrospermum Parkii subsp. Parkii (G. Don.) Hepper ou Bassia Parkii (G. Don.), Vitellaria paradoxa Gaertn [3], communément nommé "karité ", appartient à la famille des Sapotacées. C'est un arbre de (10 à 15) $\mathrm{m}$ de haut (figure 19), qui pousse à l'état spontané en savane boisée des zones sahéliennes. Le karité n'est pas cultivé mais il est protégé lors des défrichements. Son cycle végétatif est très lent. L'entrée en production n'a lieu qu'à partir de 20 ans et la pleine production se situe vers 40-50 ans [9].

En Guinée, B. Parkii est abondant dans la majeure partie de la Haute Guinée de climat soudanien et essentiellement dans les préfectures de Kankan (excepté le sud ouest de la préfecture, vers Kissidougou), Mandiana, Siguiri et Dinguiraye. L'arbre existe aussi dans le nord et le nord ouest

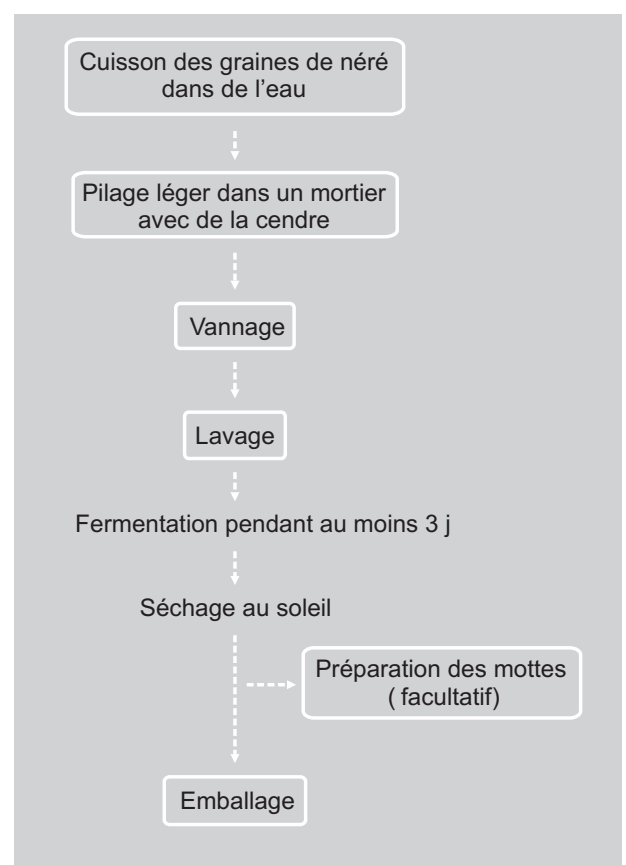

Figure 18.

Technologie de préparation du soumbara à base de graines de néré extraites des gousses de Parkia biglobosa (Guinée).

des préfectures de Kouroussa et Dabola. En Moyenne Guinée, il est présent dans les préfectures de Tougué, Koubia (est et nord-est), Mali et Koundara (figure 20).

La période de récolte du karité se situe de mai à juillet. Un arbre peut produire en moyenne (15 à 20) kg de fruits par an, soit (3 à 4) $\mathrm{kg}$ d'amandes sèches [9]. La pulpe verdâtre et sucrée, contenue dans le péricarpe du fruit mûr, est consommée parfois par les populations des zones de production. Les épluchures du fruit servent

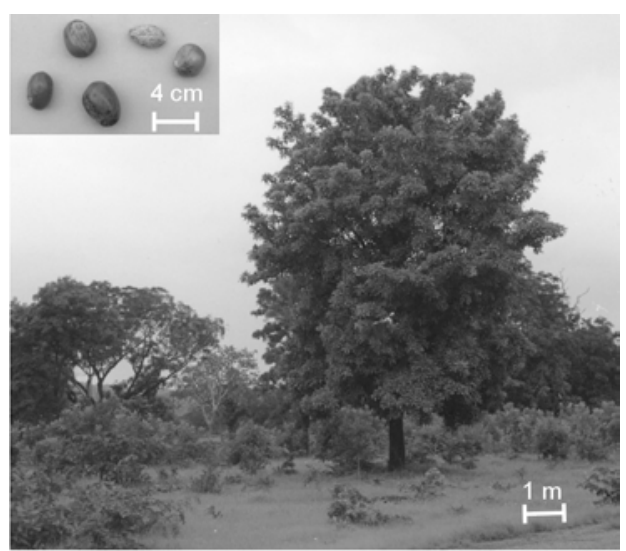

Figure 19.

Vitellaria paradoxa : arbre et graines (noix de karité). 


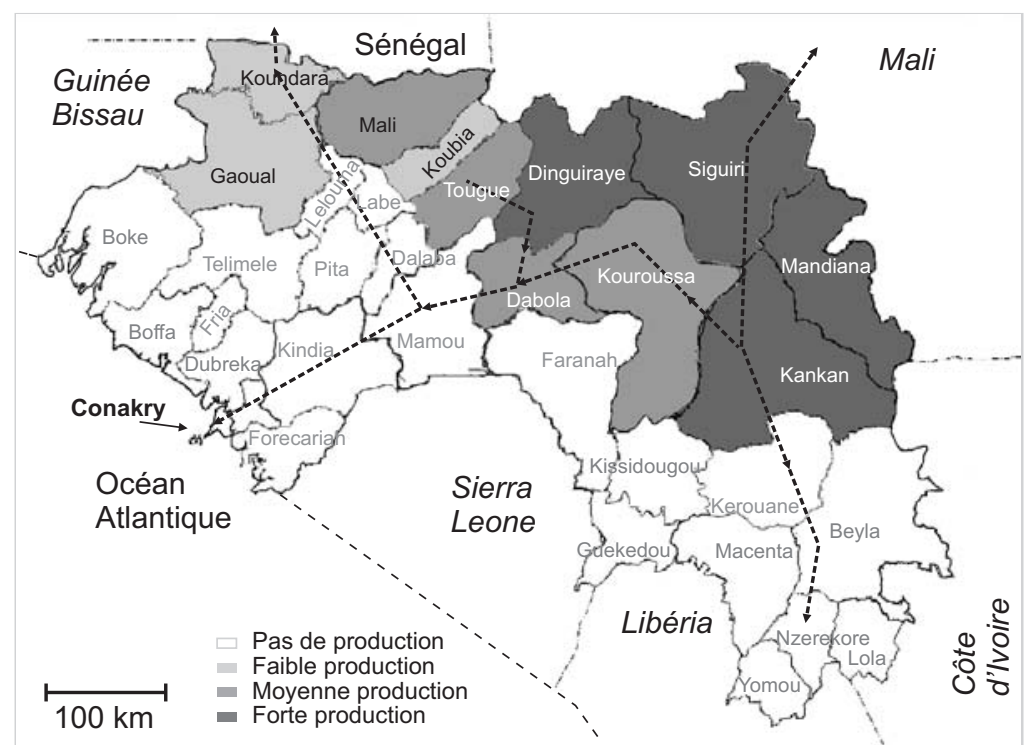

Figure 20.

Carte des zones de production de Vitellaria paradoxa ou karité en Guinée et flux commerciaux de son produit, le beurre de karité.

d'engrais dans les jardins de case. L'amande contenue dans la noix permet d'extraire le beurre de karité très utilisé dans l'alimentation, la pharmacopée, la saponification et en industries cosmétiques.

\subsection{Anisophyllea laurina $\mathrm{R}$. Br.}

De la famille des Anisophylleaceae, Anisophyllea laurina $\mathrm{R}$. Br. [5], le pommier à singe ou cantini, est un arbre qui pousse dans les galeries forestières. Les fruits de $3 \mathrm{~cm}$ de long sont jaunes à maturité, avec une pulpe juteuse très acidulée. A. laurina est connu dans toute la Guinée et il existe en grande quantité à Kindia. La fructification a lieu de mars à juin et, sur les marchés, le prix des fruits, vendus en tas, varie de $(0,07$ à 0,17$) € \cdot \mathrm{kg}^{-1}$ (figure 4$)$.

Ces fruits sont consommés crus à cause de leur pulpe très acide, ou cuits pour préparer une boisson fraîche à partir de leur eau de cuisson. Durant la période de récolte, sur les marchés de Kindia et de Conakry, cette boisson est vendue en sachets contenant chacun un fruit cuit, sous le nom de "kanti bonbon". Cette production est très développée en Haute Guinée. Elle se fait par une technique d'extraction très artisanale (figure 21) dont le rendement est de l'ordre de (13 à 15) $\mathrm{kg}$ de beurre de karité pour $100 \mathrm{~kg}$ d'amandes. En Afrique de l'ouest, les différentes étapes de la technique ont tendance à être de plus en plus mécanisées, ce qui, tout en allégeant le travail des femmes, permet d'élever le rendement de la production du beurre de karité, favorisant ainsi le développement. La plantation du karité serait à conseiller vivement et cela d'autant plus que l'espèce pousse très lentement.

Le beurre de karité produit en Haute Guinée est vendu et utilisé partout en Guinée en proportions variables. Pour cette commercialisation, le produit est enveloppé par quantité de (3 à 15) kg dans plusieurs larges feuilles de Cola cordifolia bien attachées pour les besoins du transport.

Dans les marchés de Kindia et de Conakry, le prix du beurre de karité varie entre $(0,55$ et 1,56$) € \cdot \mathrm{kg}^{-1}$ selon les périodes de l'année (figure 2).

\section{Conclusion}

Les fruits de cueillette consommés et commercialisés sur les marchés guinéens sont nombreux et très variés. Ils poussent dans toutes les régions de Guinée à des périodes bien déterminées de l'année.

Pour une grande part de la population, ils représentent un complément alimentaire, un apport vitaminique et une source de revenu. Ils sont vendus aussi bien à l'intérieur du pays que dans certains marchés de Conakry, sa capitale.

Les prix du marché Madina de Conakry sont souvent inférieurs à ceux du marché Yenguéma de Kindia. Ce décalage s'explique par le fait que la majeure partie des productions agricoles, comme les fruits de cueillette, récoltées dans toute la Guinée alimentent directement Conakry où elles peuvent être rapidement vendues ou être exportées. L'abondance des produits ainsi commercialisés à Conakry favorise l'observation de prix plus bas que ceux pratiqués à Kindia sur les mêmes produits.

Pour toutes ces raisons et compte tenu de l'intense utilisation de certains produits 
de cueillette (néré, karité, palmier à huile, tamarin, poivre noir de Guinée, etc.), il serait important que ces productions soient intensifiées par une meilleure protection des arbres et par leur intégration dans les vergers familiaux à envisager dans le respect des zones propices à ces cultures. Pour réaliser un tel projet, il serait nécessaire d'entreprendre des travaux de recherche permettant d'étudier le comportement végétatif des espèces les plus importantes et leur multiplication. Il serait alors possible de les proposer aux producteurs. L'intégration de certaines de ces espèces dans des systèmes de production agricole est l'un des objectifs de l'agriforesterie, fixés en particulier sous l'impulsion du Centre international pour la recherche en agriforesterie (ICRAF).

En Guinée, la transformation des produits de cueillette est le propre des femmes. Les procédés et l'utilisation des produits sont divers et nécessitent parfois de gros efforts physiques et beaucoup de temps de travail. La mécanisation de certaines étapes faciliterait le processus de transformation et augmenterait le revenu des populations rurales. Des recherches sur ce thème sont en cours à l'Institut de recherches agronomiques de Guinée (Irag). Elles visent à intensifier les connaissances sur les méthodes de transformation artisanales et à améliorer les techniques de valorisation des produits de cueillette. C'est dans ce cadre que le programme "Technologies" de Foulaya (Kindia, Guinée) a mis à la disposition d'un village une machine pour l'extraction de l'huile de palme et que le programme de "Conservation phytogénétique " a planté, pour observation, quelques espèces fruitières comme Saba senegalensis dans ses parcelles d'expérimentation.

\section{Références}

[1] Devey M., La Guinée, Édition Karthala, Paris, France, 1997.

[2] Bah M.A., La République de Guinée, document de télé-enseignement, Conakry, Guinée, 1974, pp. 29 et 64 .

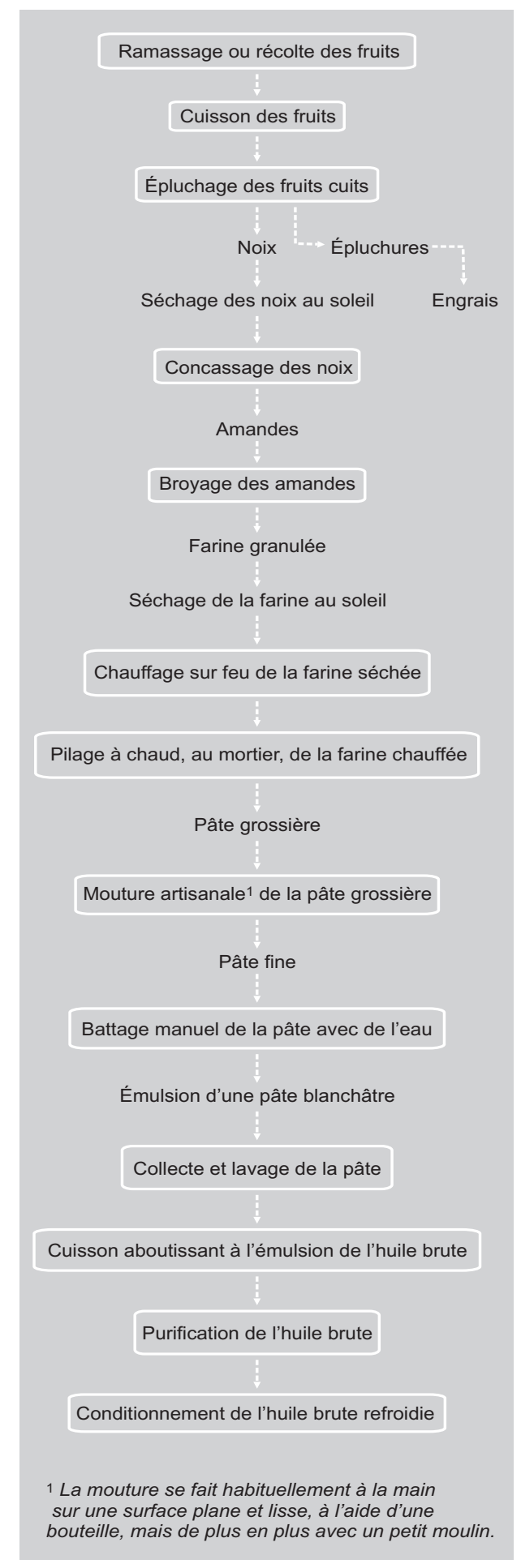

Figure 21.

Technologie artisanale d'extraction du beurre de karité issu des graines de Vitellaria paradoxa, en Guinée.
[3] Vivien J., Faure J.J., Fruitiers sauvages d'Afrique, espèces du Cameroun, Édition Guila-Kérou, France, 1996. 
[4] Lauga S.C., Le cercle des haies, paysages des agroéleveurs peuls du Fouta Djallon (plaines de Timbi, Guinée), Vol. II, C. BlancPamard (coordinatrice), Paris X, Nanterre, France, 1997.

[5] Baumer M., Arbres, arbustes et arbrisseaux nourriciers en Afrique occidentale, EndaÉditions, série Études et recherches, Dakar, Sénégal, 1995.

[6] Basilevskaia V., Plantes médicinales de Guinée, Impr. Patrice Lumumba, Conakry, Guinée, 1971.
[7] Fanshawe D.B., Fifty common trees of Zambia, Min. nat. res. tourism, Bull. $n^{\circ} 5$, Luzaka, Zambia, 1968.

[8] Arbonnier M., Arbres, arbustes et lianes des zones sèches d'Afrique de l'Ouest, Cirad / MNHN /UICN, Mauguio, France, 2000.

[9] Anonyme, Mémento de l'agronome, Ministère de la Coopération et du développement, $4^{\mathrm{e}}$ éd., Collection Techniques rurales en Afrique, St-Just-La Pendue, France, 1991.

\section{Principales frutos de recolección consumidos y comercializados en Guinea.}

Resumen - Introducción. País de África occidental, Guinea tiene un clima tropical y un relieve accidentado con una importante red hidrográfica. Presenta cuatro regiones naturales en las que se desarrollan espontáneamente varias especies frutales cuyos frutos son consumidos y vendidos en los mercados nacionales. Para explotar mejor dichos productos, se ha intentado, mediante encuestas, elaborar una base de datos fiable que identifique las especies frutales implicadas, sus zonas y períodos de reproducción, el canal de comercialización de los productos y sus técnicas de conservación, transformación y consumo. Material y métodos. Se efectuó, en dos mercados de guinea y de marzo de 1998 a febrero de 2000, un seguimiento del precio de frutos de recolección comercializados y un análisis detallado de su producción y utilización. Para ello, se recopiló la información necesaria (precio, período, acondicionamiento, canal de comercialización) mediante encuestas realizadas cada quince días y se apuntaron estos datos en una ficha específica. Resultados. El estudio puso de relieve posibles problemas de suministro de mercados, relacionados con las zonas de producción. Entre las especies identificadas, doce se revelaron más importantes y fueron objeto de análisis. Se definieron sus zonas de producción. La mayoría de la fruta comercializada en los mercados investigados madura entre marzo y junio; representando una fuente de ingresos para una buena parte de la población. Se catalogaron las técnicas de conservación, comercialización, transformación y consumo. Conclusión. La investigación efectuada conduce a favorecer la protección de los frutales de recolección y a aconsejar su introducción progresiva en los huertos familiares. Los programas de investigación nacionales también están intentando intervenir en la transformación y conservación de los productos procedentes de recolección, que plantean grandes dificultades a los productores.

Guinea / especies endémicas / frutales / recolección / localización de la producción / precios de mercado / usos 